\title{
Thermal conductivity measurements of road construction materials in frozen and unfrozen state
}

\author{
Norbert I. Kömle $\cdot$ Hui Bing $\cdot$ Wen Jie Feng · Roman Wawrzaszek \\ Erika S. Hütter · Ping He · Wojciech Marczewski · Borys Dabrowski · \\ Kathrin Schröer · Tilman Spohn
}

Received: 5 January 2007/ Accepted: 16 May 2007/Published online: 11 July 2007

(C) Springer-Verlag 2007

\begin{abstract}
A series of thermal conductivity measurements for various materials was performed in a large climate chamber. The size of the chamber allowed the preparation of relatively large samples in a controlled thermal environment. Three types of thermal sensors were used: (1) two needle probes; (2) a grid of temperature sensors, evenly distributed inside the sample; (3) two additional thermal probes, which were simplified versions of an instrument originally developed for measuring thermal properties of the ice/dust mixture expected to exist at the surface of a comet nucleus. They consist of a series of individual temperature sensors integrated into a glass fibre rod. Each of these sensors can be operated in an active (heated) or passive (only temperature sensing) mode. The following sample materials were used: fine-grained reddish sand, coarse-grained moist sand, gravels with various grain size distributions from $<1 \mathrm{~cm}$ up to about $6 \mathrm{~cm}$, and for
\end{abstract}

Presented at the 1st Asian Conference on Permafrost (ACOP), Lanzhou, Gansu, China, 7-9 August 2006

N. I. Kömle $(\bowtie) \cdot$ E. S. Hütter

Space Research Institute, Austrian Academy of Sciences,

Schmiedlstrasse 6, $8042 \mathrm{Graz}$, Austria

e-mail: norbert.koemle@oeaw.ac.at

H. Bing · W. J. Feng · P. He

Cold and Arid Regions Environmental and Engineering

Research Institute (CAREERI), Chinese Academy of Sciences,

Lanzhou, China

R. Wawrzaszek · W. Marczewski · B. Dabrowski

Space Research Centre (SRC), Polish Academy of Sciences,

Warsaw, Poland

K. Schröer · T. Spohn

Institut für Planetologie (IFP), Universität Münster,

Münster, Germany comparison and calibration pure water (with convection suppressed by adding agar-agar), compact ice, and compact granite. Of particular interest are the measurements with composite samples, like stones embedded in an agar-agar matrix. We describe the evaluation methods and present the results of the thermal conductivity measurements.

Keywords Gravel · Permafrost · Sand · Thermal conductivity

\section{Introduction}

The experiments reported in this paper served a twofold purpose. First, in many physical and engineering problems the thermal conductivity of the materials is a key parameter controlling to a high extent the thermo-physical behaviour of the system. For example, in the construction of road and railway routes the distribution of artificially or naturally generated heat in the underground determines the stability of the bed and thus the safety of pathways. This is particularly important on seasonally or permanently frozen ground, like the areas transected by the Qinghai-Tibet Railway [14, 15].

The second aspect of our paper deals with the development of suitable sensors for thermal conductivity measurements itself. For our measurements we have used, among else, so-called needle probes. They belong to the category of transient or non steady state measurement techniques. In general, when using a transient measurement procedure, an unsteady temperature gradient is induced inside the sample by a constant heat source. The heating occurs over an adequate time period. The thermal conductivity and/or diffusivity can be derived from the measured temperature response due to heating. Common 
commercialized techniques using this principle are needle probes, hot wire methods, and the hot strip method. A comprehensive summary of available transient as well as steady state methods can be found, e.g. on the evitherm website [5]. The advantages of transient measurement techniques as compared to steady state techniques (like the hot plate method) are (1) shorter measurement periods, (2) suitability for a wide range of materials (solid and granular) and (3) a lower technical complexity. The latter property makes them more suitable for field applications or even application in space missions. On the other hand, the measurement accuracy of transient methods is usually lower than that of steady state methods. Therefore, steady state methods may be more useful for calibration purposes.

Interpretation of needle probe measurements in terms of thermal conductivity is most simple if the sample to be measured has a homogeneous structure, is originally isothermal, and is large enough that boundary effects can be excluded. However, under more complex conditions, which frequently occur in the natural environment, evaluation of thermal conductivity from measured temperature data needs great care. We will consider an appropriate reduction method for the raw data in order to derive reliable conductivity values from measured raw data.

Another way to evaluate thermal conductivity of a medium indirectly is to use a grid of temperature sensors, e.g. RTDs $^{1}$ distributed across the sample and to introduce a vertical temperature gradient. One way to achieve this is to bring the bottom side of the sample in thermal contact with a cooling plate. In our tests, such a method was used to compliment and cross-check the needle probe measurements.

In many applications the variation of thermal properties with depth is of interest. This cannot be easily achieved by standard needle probes like the Hukseflux TP02 [7]. They can only provide average values over the length of the probe. However, a thermal probe developed in the recent years in the frame of ESA's ${ }^{2}$ comet mission Rosetta has some ability to measure the variation of thermal properties with depth in a soil. This instrument $\left(\right.$ MUPUS $\left.^{3}\right)$, being a part of the payload of the Rosetta Lander Philae, is currently on the way to its target comet Churyumov-Gerasimenko. After landing on the comet nucleus' surface, the thermal properties of the cometary near-surface ice will be derived [1, 8]. The probe consists of several segments which can be separately heated in order to measure the thermal properties of different layers subsequently. The concept of MUPUS may also be useful for applications in

\footnotetext{
1 Resistance Temperature Device, see http://www.temperatureworld.com/rtd.htm

${ }^{2}$ European Space Agency

${ }^{3}$ MUlti-PUrpose Sensors for surface and sub-surface science
}

terrestrial environments. Therefore several probes of a simplified variant named EXTASE ${ }^{4}$ have been built in the frame of another project [10,13]. First tests showed that the interpretation of temperature data recorded by the EXTASE probe in terms of thermal conductivity is by no means an easy task. It demands a rather detailed thermal model of the sensor itself and calibration measurements in various well-known materials. In order to support this goal and to provide a broader basis for the interpretation of the expected MUPUS measurements at the comet surface, we have done some parallel tests with the EXTASE probes and the needle probes in a well-known medium and compared the results in terms of the obtained thermal conductivity values.

\section{Experimental equipment and setup}

\subsection{Climate chamber and sample container}

The tests were performed in a thermally controlled environment, namely a climate chamber of the CAREERI (Lanzhou, China). This climate chamber allowed to establish a homogeneous temperature environment in the range of $-40^{\circ} \mathrm{C}$ to $+70^{\circ} \mathrm{C}$. The interior size of the chamber is approximately a cube of $90 \mathrm{~cm}$ side length. A system of fans permits fast relaxation of temperature inside the chamber to a prescribed value. Furthermore, air humidity inside the chamber can be controlled externally and set to a prescribed value. During operation, the power devices of the chamber are cooled by a cold water circuit.

The second major device used in our experiments is the sample container. It is of rectangular shape $(70 \mathrm{~cm} \times$ $70 \mathrm{~cm} \times 35 \mathrm{~cm})$ and its sides are thermally insulated. The bottom is connected to a cooling system working with alcohol as cooling agent. This setup allows us to establish a constant temperature at the bottom of the sample container, which may differ from the set chamber temperature. Thus, it can be used to establish a vertical temperature gradient inside a test sample.

\subsection{Thermal conductivity sensors}

Three different methods for determining thermal conductivity were applied: (1) A commercial needle probe (Hukseflux TP02); (2) a grid of individual temperature sensors (platinum RTDs) which measures the temperature variations inside the sample during cooling periods; (3) a custom-built sequentially working probe (EXTASE) for

\footnotetext{
${ }^{4}$ EXperimental Thermal probe for Applications in Snow research and Earth sciences
} 
measuring the vertical temperature profile and the local thermal diffusivity.

In the first case, thermal conductivity is obtained by plotting the measured probe temperature versus the natural logarithm of time [2]. However, under non-ideal conditions a careful reduction of the raw data is necessary, as will be discussed in more detail below. The other two applied methods demand more extensive thermal modeling. The temperatures obtained from the probe measurements $(\mathrm{Hu}-$ kseflux TP02, EXTASE) result from a heat flux introduced in radial direction by sensor heating. By contrast, the values measured by the RTD temperature sensors are mainly due to an axial (vertical) heat flow caused by bottom cooling of the sample. Furthermore, it should be noted that the grid of RTD sensors measures the three-dimensional temperature distribution, although with a coarse resolution. All three types of sensors, inserted into a sand sample, can be seen in Fig. 1. Figure 2 shows the build-up of the Hukseflux TP02 probe in more detail.

The needle probes give straightforward results if the sample has homogeneous structure, a good contact to the sensor, and is initially isothermal and in a thermal equilibrium state. However, large temperature gradients over the length of the sensor can cause significant measurement errors. The sensor needle of the Hukseflux TP02 probe has a length of $150 \mathrm{~mm}$ and a diameter of $1.5 \mathrm{~mm}$. It consists of a heating wire with a heater resistance of $75.52 \Omega / \mathrm{m}$ and two thermocouple junctions for differential temperature measurement. The sensors and the heating wire are embedded in a completely sealed stainless steel housing.

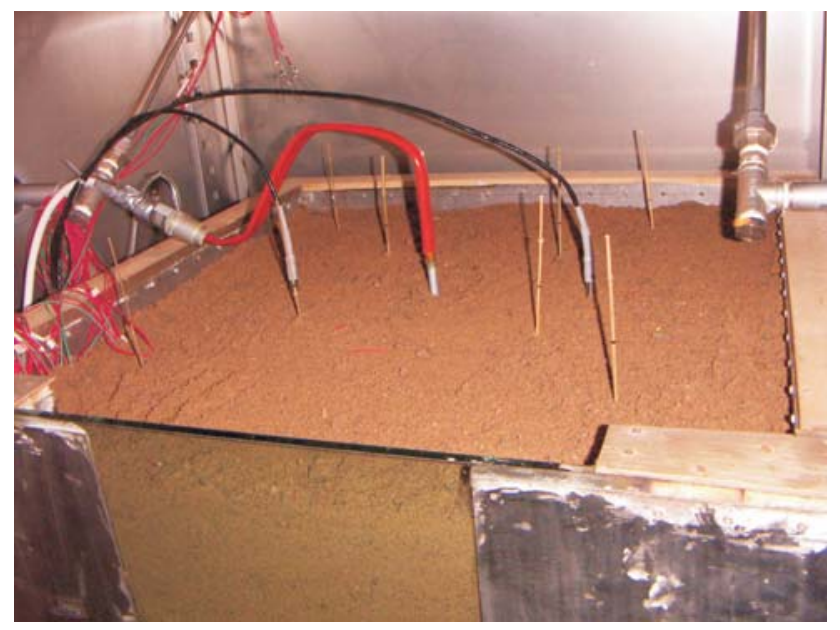

Fig. 1 Thermal probes used for determining heat conductivity and temperature profiles. In the center, one EXTASE probe is visible, to the left and to the right of it the Hukseflux TP02 sensors are inserted into the soil. The wooden rods mark the positions of the RTD sensors, which are mounted vertically along the rods with about $5 \mathrm{~cm}$ mutual distance
The EXTASE probe consist of a $32 \mathrm{~cm}$ long rod composed of glass fiber, which contains a series of heater foils simultaneously acting as temperature sensors $[8,11]$. Sixteen individual sensors (with increasing length from top to bottom) are attached to the interior side of the glass fiber structure. The temperature response of each sensor to heating by a known electrical power is linked to the thermal properties of the surrounding medium and can, therefore, be used to evaluate the local thermal diffusivity.

\subsection{Sample materials and experiments}

A standard material used for calibration purposes is agaragar in a temperature range from 0 to $20^{\circ} \mathrm{C}$ [7]. It was chosen for calibration because it has exactly the thermal conductivity of pure water as given in many literature sources. However, due to its jelly-like state, it is insensitive against thermal convection, while still providing an excellent thermal contact with the sensor. Upon freezing it was found that the thermal conductivity is the same as that of regular compact ice.

From the materials used in road/railway construction along the Qinghai-Tibet route, we have selected the following samples: big granite stones (in order to find out the conductivity of compact granite), big (ca. $6 \mathrm{~cm}$ sized) broken gravel of the same granite, rounded river gravels with various grain sizes, dry red dune sand, and slightly wetted sand. In order to evaluate the conductivity of composite samples, we have also investigated a mixture of big gravels and agar-agar. Most materials were measured at different temperature levels and vertical temperature gradients. The samples with inserted sensors are shown in Figures 3 and 4.

\section{Data evaluation}

\subsection{Conductivity of composite materials}

The effective thermal conductivity of composite materials (e.g. rubble piles filled up with some finer material) has been a controversial field since long. Various formulas have been proposed, the predictions of which sometimes deviate widely. A common aspect is the idea that the effective conductivity is calculated analogous to electrical circuits, namely a combination of parallel and series connections of the conductivities of the single components. Our experiments with granite stones, agar, and composite samples (stones + air, stones + agar) offer the possibility to test some simple formulas predicting the conductivity of two-component samples practically.

As an example consider the measurements in the gravel sample filled by agar-agar. The volume ratio filled with the 


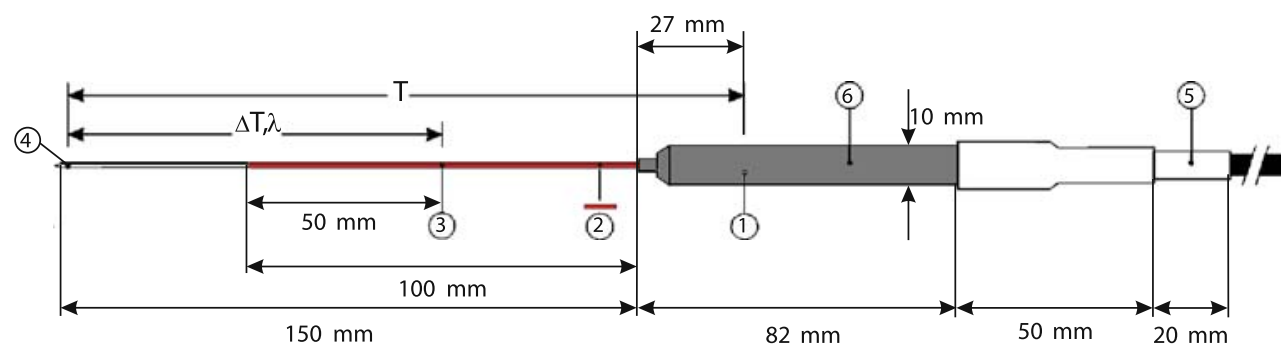

Fig. 2 Dimensions and build-up of the Hukseflux TP02 thermal conductivity probes used to obtain the thermal conductivity data. The sensors including the needle are hermetically sealed in a stainless steel casing, which makes them suitable also for vacuum applications

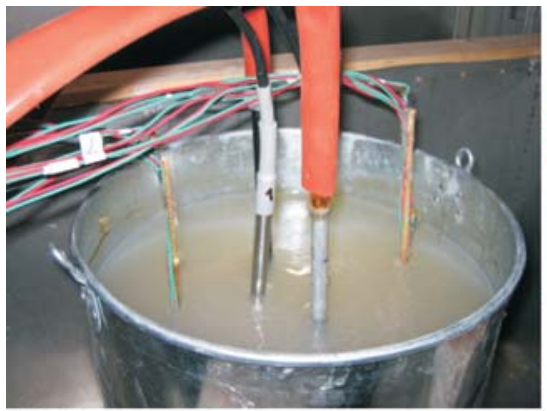

agar

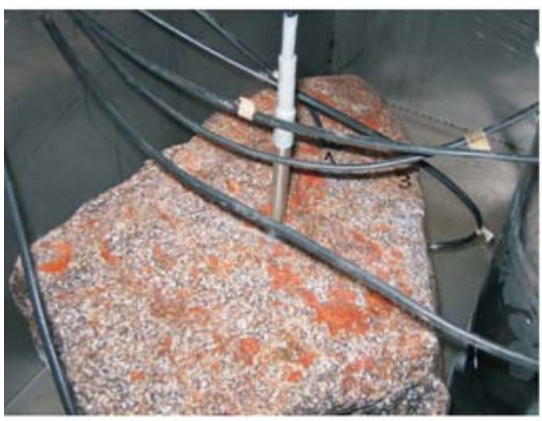

granite

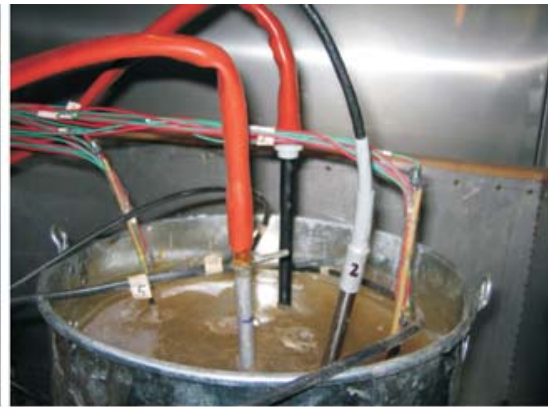

agar-ice

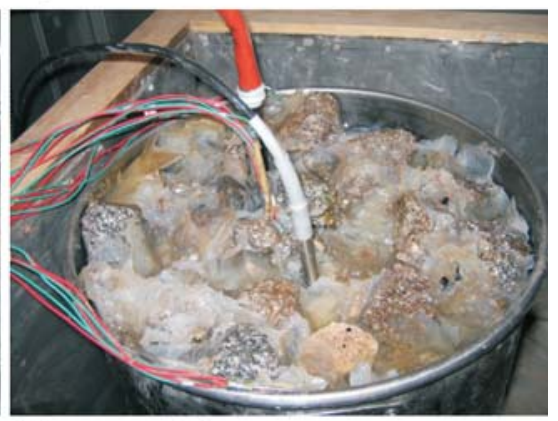

big gravel + agar

Fig. 3 Sample materials with inserted sensors. Upper left: the agar-agar gel used as a calibration standard for the hot wire sensors; upper right: ice produced by freezing the agar-agar gel; lower left: a big granite stone with a Hukseflux TP02 conductivity sensor inserted into a borehole; lower right: a mixture of big granite gravel with agar-agar gel, produced by filling up the pore spaces between the single granite stones with hot agar-agar solution, which becomes solid by chilling

agar gel is denoted by $\psi$ (equivalently $\psi$ is the porosity of the stony matrix); $(1-\psi)$ represents the volume fraction filled by the stones. Then the simplest way to predict the effective conductivity of the composite sample would be

$\lambda=(1-\psi) \lambda_{\text {stone }}+\psi \lambda_{\text {agar }}$

Another formula frequently used in the literature is the socalled Russel-formula [3, 4]. It combines the porosity and the single conductivities in the following way:

$\lambda=\lambda_{\text {stone }} \frac{f \psi^{2 / 3}+1-\psi^{2 / 3}}{f\left(\psi^{2 / 3}-\psi\right)+1-\psi^{2 / 3}+\psi}$

with $f=\lambda_{\text {agar }} / \lambda_{\text {stone }}$

The porosity $\psi$ of the composite sample (respectively the fraction filled by the agar material) has been experimentally determined as $\psi=0.48$. The evaluation of 16 conductivity measurements in an agar-agar sample and in a big granite stone (see Fig. 3) gives average conductivity values of $\lambda_{\text {agar }}=0.58 \mathrm{~W} \mathrm{~m}^{-1} \mathrm{~K}^{-1}$ and $\lambda_{\text {stone }}=2.69 \mathrm{~W} \mathrm{~m}^{-1} \mathrm{~K}^{-1}$. Using formula (1) these data would give a calculated value of $\lambda=1.68 \mathrm{~W} \mathrm{~m}^{-1} \mathrm{~K}^{-1}$. This result compares favourably with the measured value (averaged from 14 single measurements) of $\lambda=1.64 \mathrm{~W} \mathrm{~m}^{-1} \mathrm{~K}^{-1}$. The Russel formula would give a slightly smaller value, namely $\lambda=$ $1.56 \mathrm{~W} \mathrm{~m}^{-1} \mathrm{~K}^{-1}$. 


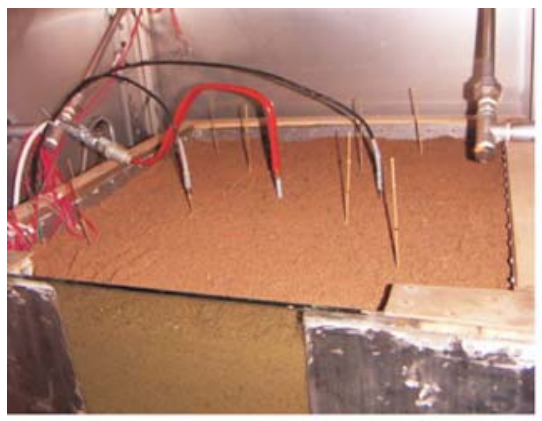

moist sand

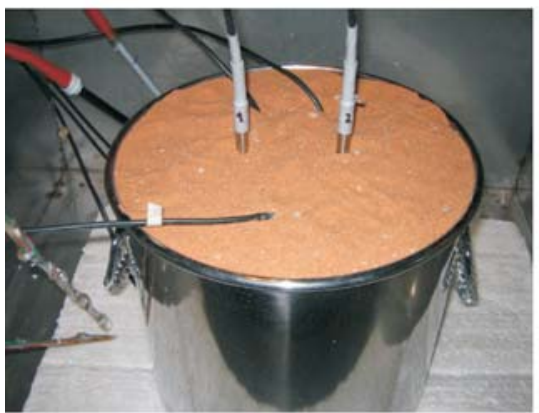

red sand

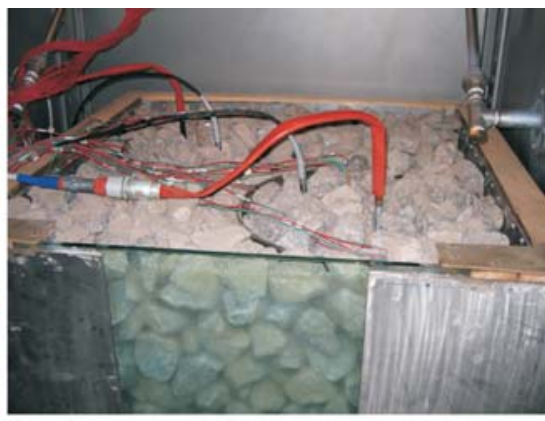

broken gravel

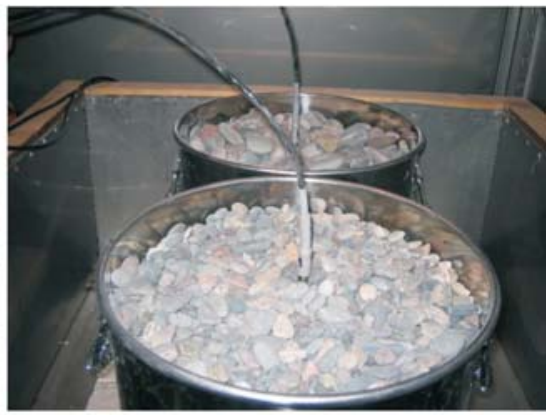

rounded gravels

Fig. 4 Sample materials with inserted sensors. Upper left: moist sand sample inside the climate chamber, with EXTASE probes, Hukseflux TP02 probes and RTD temperature sensors distributed over the surface; upper right: big granite stones gravel with a similar sensor distribution as used for the moist sand sample; lower left: dry red send from Gansu region with two Hukseflux TP02 probes and some RTDs inserted; lower right: rounded river gravels with two different size distributions: $1 \mathrm{~cm}<d<2 \mathrm{~cm}$ (front side) and $2 \mathrm{~cm}<d<4 \mathrm{~cm}$ (rear side)

\subsection{Needle probe measurements}

In the following, we describe the evaluation of the measurements with the needle probes. The derived thermal conductivity values are summarized in Table 1. For the moist sand sample, the measured thermal conductivities in different temperature ranges (unfrozen as well as frozen) are plotted in Fig. 5.

Table 1 Average values of thermal conductivity for the sample materials investigated by the HUKSEFLUX TP02 hot wire conductivity probes

\begin{tabular}{lll}
\hline Sample material & $\begin{array}{l}\text { Number of } \\
\text { measurements }\end{array}$ & $\begin{array}{l}\text { Thermal conductivity } \\
{\left[\mathrm{W} \mathrm{m}^{-1} \mathrm{~K}^{-1}\right]}\end{array}$ \\
\hline Red sand & 6 & 0.31 \\
Moist sand & & 0.82 \\
Rounded gravel 1-2 cm & & 0.40 \\
Rounded gravel 2-4 cm & & 0.70 \\
Agar & 16 & 0.58 \\
Agar-ice & & 2.10 \\
Granite (solid) & 16 & 2.68 \\
Big gravel with air & 16 & 0.80 \\
Big gravel with agar & 14 & 1.65 \\
\hline
\end{tabular}

\subsubsection{Measurements and reduction method}

The raw data produced by the TP02 needle probes give the temperature increase of the sensor needle as a function of time. According to the theory of a thin cylindrical rod heated with constant power in a homogeneous medium, the heating curve has two characteristic phases, which can be easily separated when the temperature signal is plotted on a logarithmic time scale: the initial phase is a transient, fast temperature increase, the shape of which is determined by the thermal inertia of the needle and by the contact resistance between needle and medium [2]. The second part of the signal should show a linear increase with the natural logarithm of time, counted from the onset of heating. This part of the curve is evaluated to determine the thermal conductivity of the surrounding medium according to the formula

$\lambda=\frac{Q}{4 \pi \Delta T} \ln \left(\frac{t_{2}}{t_{1}}\right)$

where $Q$ is the heater power per unit length. The thermal conductivity is calculated from temperature measurements at two different points in time $\left(t_{1}\right.$ and $\left.t_{2}\right)$ in the logarithmically linear part of the heating interval $[7,9,12] . \Delta T$ is the temperature increase of the sensor needle during the 

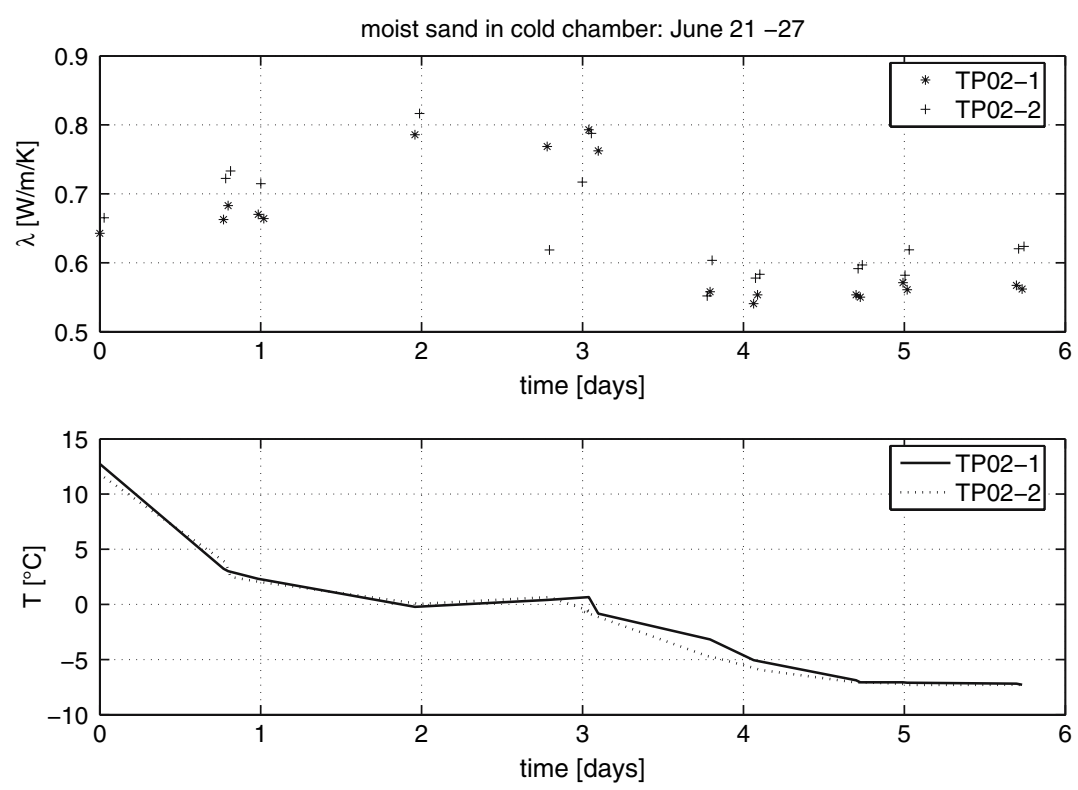

Fig. 5 Results of the thermal conductivity measurements in the moist sand sample using the Hukseflux TP02 needle probes in different temperature and moisture ranges

heating interval. Note that the result for $\lambda$ is independent of the medium properties $\rho$ and $c_{p}$ as well as of the contact resistance between medium and sensor. Only the shape of the temperature curve in the initial part of the graph and the total height of $\Delta T$ depend on the contact resistance. However, this does not affect $\lambda$, which only depends on the inclination in the rear part. Ideally the measurements should be done in an initially isothermal sample. There can be two types of disturbing influences which may cause faulty results: (1) a nonlinear temperature gradient in the sample over the length of the needle and (2) a temperature trend with time during the measuring interval, which is not caused by the active heating of the sensor needle.

The first problem is a second order effect. As long as the thermal gradient is approximately linear, it does not affect $\Delta T$, because the sensor measures the difference between the heated and the unheated part of the needle. To eliminate the second effect, a measurement always consists of two parts. In the first part the temperature variation before the onset of heating is measured, and in the second part the signal caused by sensor heating is recorded. Assuming that the trend is linear over the length of the measurement it can be eliminated from the data. The evaluation steps from the raw signal to the derived thermal conductivity value are illustrated in Fig. 6. In this example, we have used a measurement in agar-ice where a clear temperature trend was seen in the raw data. As can be seen from the graphs, the derived signals may be quite sensitive to this correction.

Another item that must be carefully observed in the evaluation of the signals is the possible influence of the boundary conditions. This is especially important in media with high conductivity and for measurements in small samples, where the heat wave triggered by the sensor may reach the boundary of the sample before the measurement is completed. In this case the derived conductivity value may be too high if the last part of the signal is included in the evaluation. Therefore, the part of the signal potentially influenced by boundary effects should not be used. In addition, for every individual measurement the graph on the logarithmic time scale should also be visually inspected to identify the linear part.

To estimate the length of the transient part of the signal the following formula can be used:

$t_{\text {transient }}=\frac{10 D^{2}}{\kappa}$

Hereby $D$ is the diameter of the heated needle and $\kappa=\frac{\lambda}{\rho c_{p}}$ is the thermal diffusivity of the surrounding medium.

\subsection{RTD temperature measurements}

Another way to evaluate the thermal conductivity of the samples is to use the readings of the RTD temperature sensors during the extended cooling phases of the chamber and the bottom of the sample container. In the following, we analyse the cooling phases of the unfrozen and frozen sand sample and the gravel sample with the help of a simple thermal model. Hereby, we compare the measured temperature profiles along vertical lines (indicated in Figures 7 and 8) with the model results. 

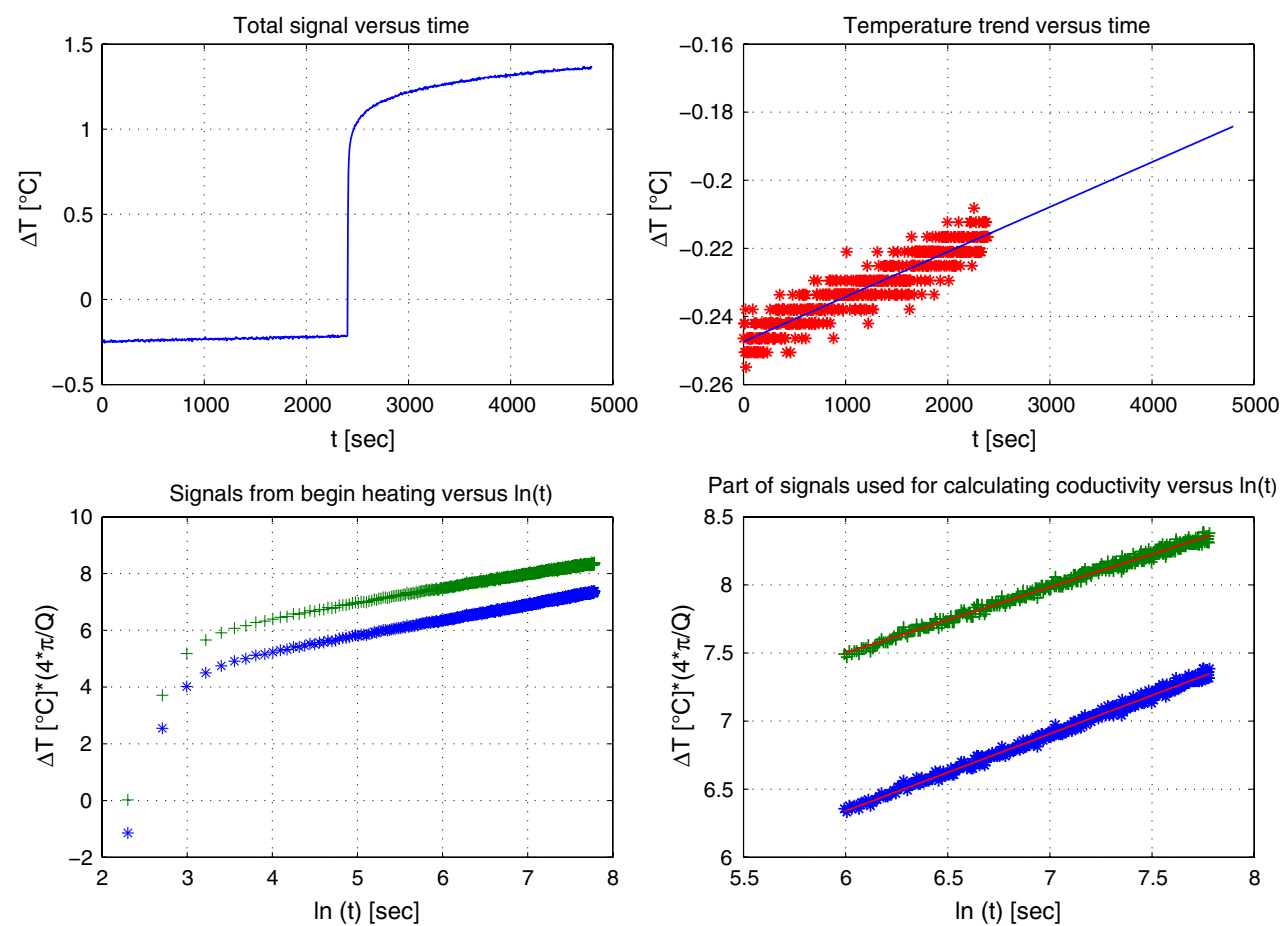

Fig. 6 Different steps of signal analysis for evaluating the thermal conductivity from the measured temperature signal of a needle probe. Upper left: measured temperature increase in response to sensor heating; upper right: evaluation of the general temperature trend in the sample before heating; lower left: corrected (green) and uncorrected (blue) temperature increase in response to sensor heating over a logarithmic time coordinate; only the heated part of the signal is plotted here; lower right: part of the signal used for the evaluation of the thermal conductivity

The initial conditions are taken from the measured vertical profile at time $t=0 \mathrm{~h}$. As the lower and upper boundary conditions of the problem, the temperature variations measured by the sensors positioned closest to the upper and lower sample surface are used. Subsequently, a one-dimensional thermal model is applied to each of the vertical temperature profiles, assuming that the lateral heat

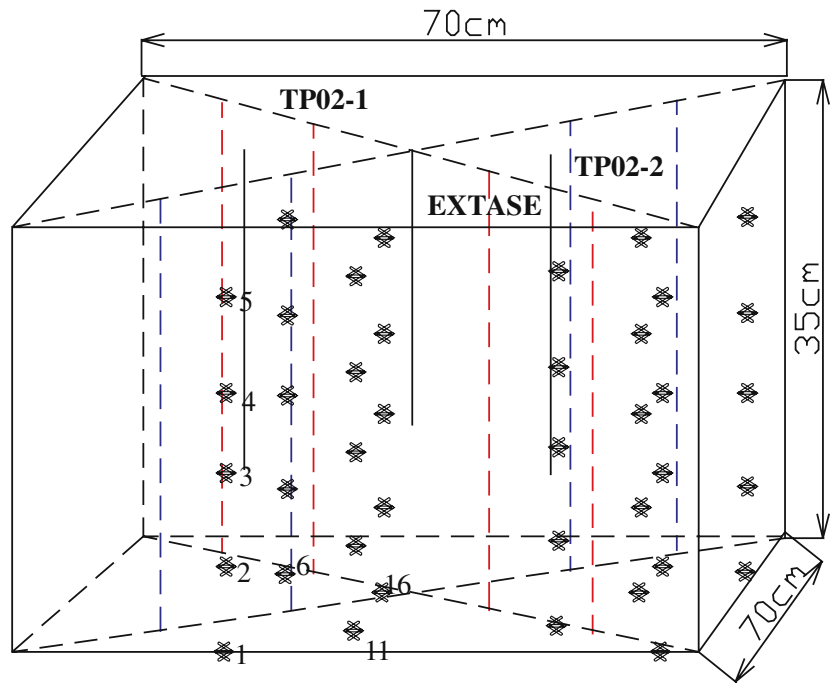

Fig. 7 Positions of RTD-sensors inside moist sand sample flows can be neglected. This assumption is justified since the side walls of the sample container are well isolated. Thus cooling should take place primarily due to the contact of the medium with the actively cooled bottom plate and by the exposure of the sample surface to the cold air inside the climate chamber. The input parameters for the thermal model are density $\rho$, heat capacity $c_{p}$ and thermal conductivity $\lambda$. The modelling was performed over a time interval of $20 \mathrm{~h}$ (for the sand sample) and $8 \mathrm{~h}$ (for the gravel sample). Finally the modelling results are compared

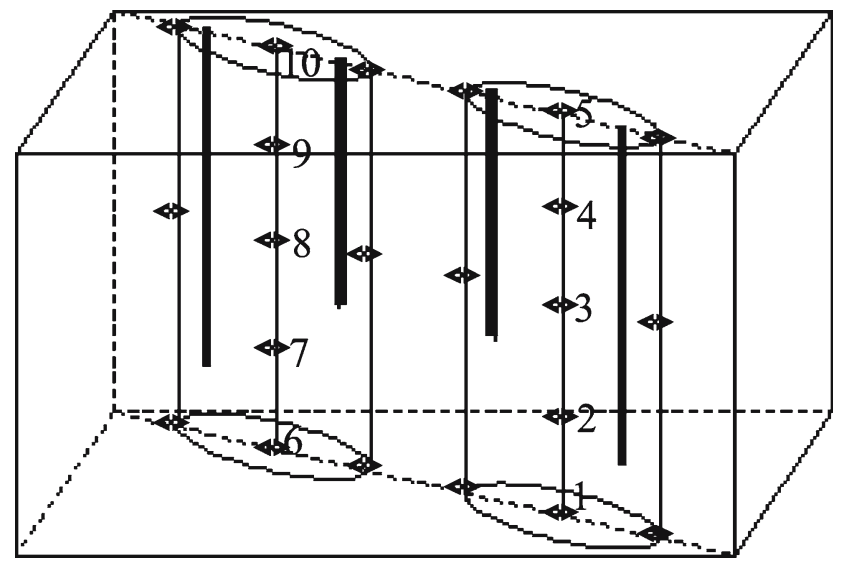

Fig. 8 Positions of RTD-sensors inside gravel sample 
with the temperatures measured by the sensors placed between the bottom and the top sensor.

\subsubsection{Moist sand sample}

The average density of the moist sample was experimentally determined as $\rho=1660 \mathrm{~kg} \mathrm{~m}^{-3}$. The heat capacity was taken from literature references [6] as $c_{p}=710 \mathrm{~J} \mathrm{~kg}^{-1}$. Having constrained the other two parameters in this way, we can vary the thermal conductivity $\lambda$ in order to obtain a reasonable fit to the measured temperature profiles. The result of this procedure is shown in Fig. 9 for the sand sample in the positive temperature range and in Fig. 10 for the frozen sand sample.

It turns out that with $\lambda=0.8 \mathrm{~W} \mathrm{~m}^{-1} \mathrm{~K}^{-1}$ in the positive temperature range and $\lambda=0.6 \mathrm{~W} \mathrm{~m}^{-1} \mathrm{~K}^{-1}$ in the negative temperature range, we obtain very good coincidence with the measured temperatures along the four vertical profiles. Only the left front profiles (denoted as "Profile 1" in the figures) show a slight deviation, which may be due to locally inhomogeneous sample structure or deviations from the assumed one-dimensional geometry. The best fit values obtained for $\lambda$ are well within the range measured by the needle probes, as can be seen by comparison with the plot shown in Fig. 5.

\subsubsection{Gravel sample}

A similar analysis was performed for the gravel sample. Hereby, it should be noted that in this case the measurements made by the needle probes are outside the range for which the probes are specified to guarantee accurate measurements. The contact of the sensor needles to the surrounding blocky stones is poor and the grain size of the stones is only a few times smaller than the needle length. Moreover, it cannot be excluded that air convection in the large pores between the stones influence the results. Nevertheless, it was possible to make a large amount of measurements over a number of days under conditions when the cooling fans of the chamber were switched off temporarily and thus no forced convection took place in the chamber. From the inspection of the graphs it turned out that they had a clearly linear part which could be used to derive a value for the thermal conductivity in a rather narrow range around $\lambda=$ $0.8 \mathrm{~W} \mathrm{~m}^{-1} \mathrm{~K}^{-1}$. The measured and calculated temperature curves along the vertical paths indicated in Fig. 8 are shown in Fig. 11.

The measured temperature variations match the ones calculated with $\lambda=0.8 \mathrm{~W} \mathrm{~m}^{-1} \mathrm{~K}^{-1}$ quite well in the first few hours of the cooling phase. Later on deviations in-
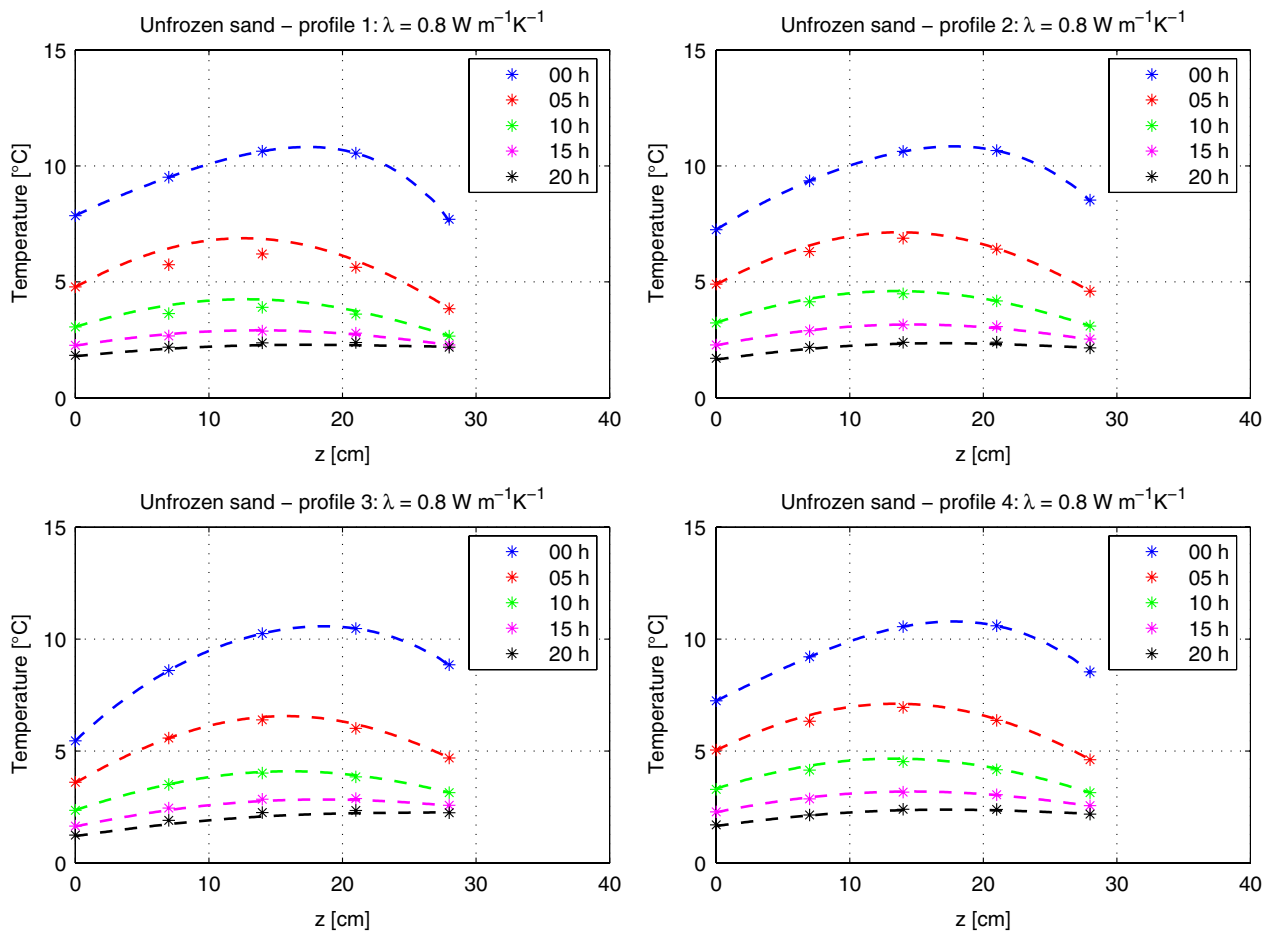

Fig. 9 Fitting of the measured temperature profiles inside the unfrozen moist sand sample by a heat conduction model. The four panels correspond to the temperature profile measured along the vertical paths indicated in Fig. 7. $z=0 \mathrm{~cm}$ corresponds to the bottom of the sample, $z=28 \mathrm{~cm}$ is the position of the uppermost sensors, which are placed a few $\mathrm{cm}$ below the sample surface 

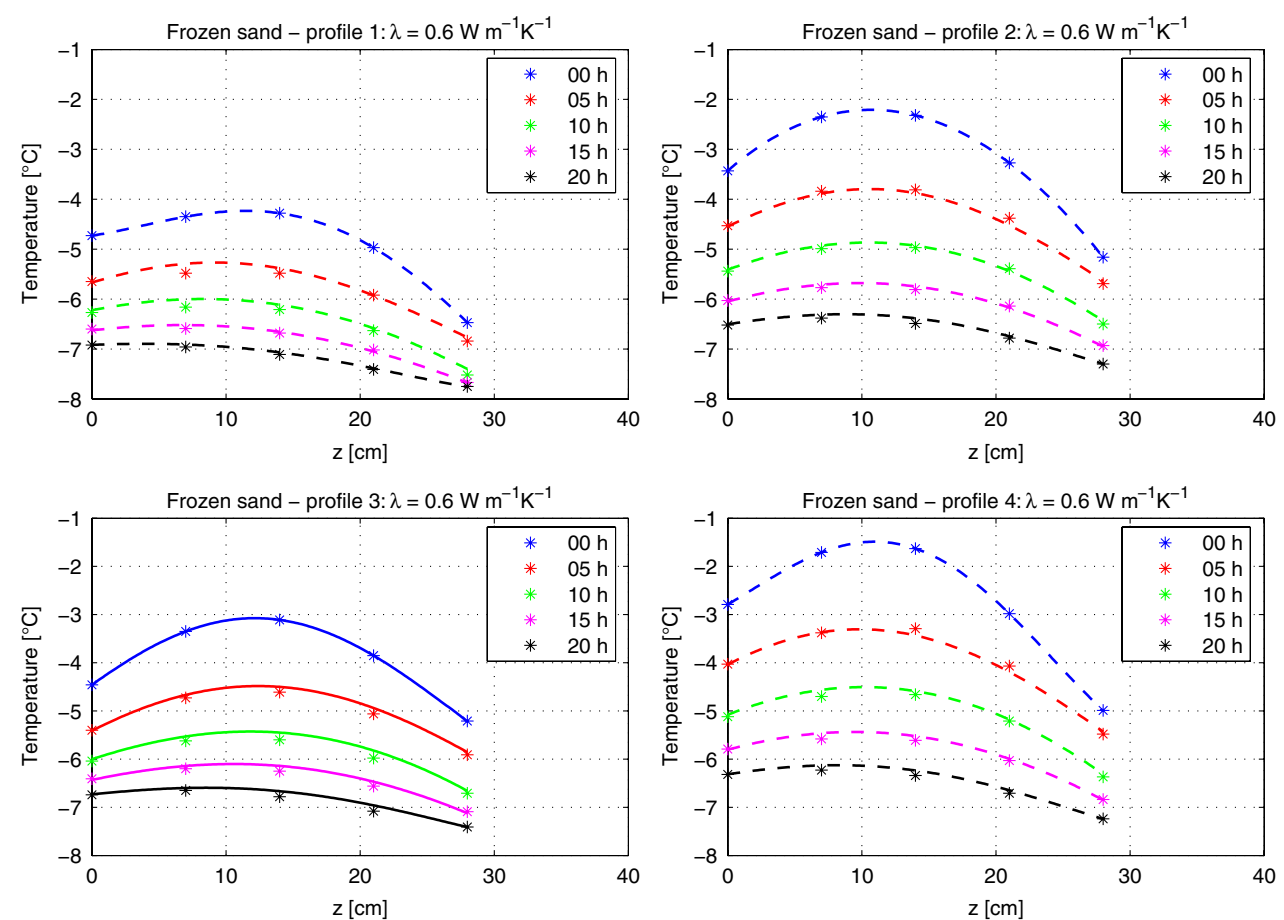

Fig. 10 Fitting of the measured temperature profiles inside the frozen moist sand sample by a heat conduction model. The four panels correspond to the temperature profile measured along the vertical paths indicated in Fig. 7. $z=0 \mathrm{~cm}$ corresponds to the bottom of the sample, $z=28 \mathrm{~cm}$ is the position of the uppermost sensors, which are placed a few $\mathrm{cm}$ below the sample surface
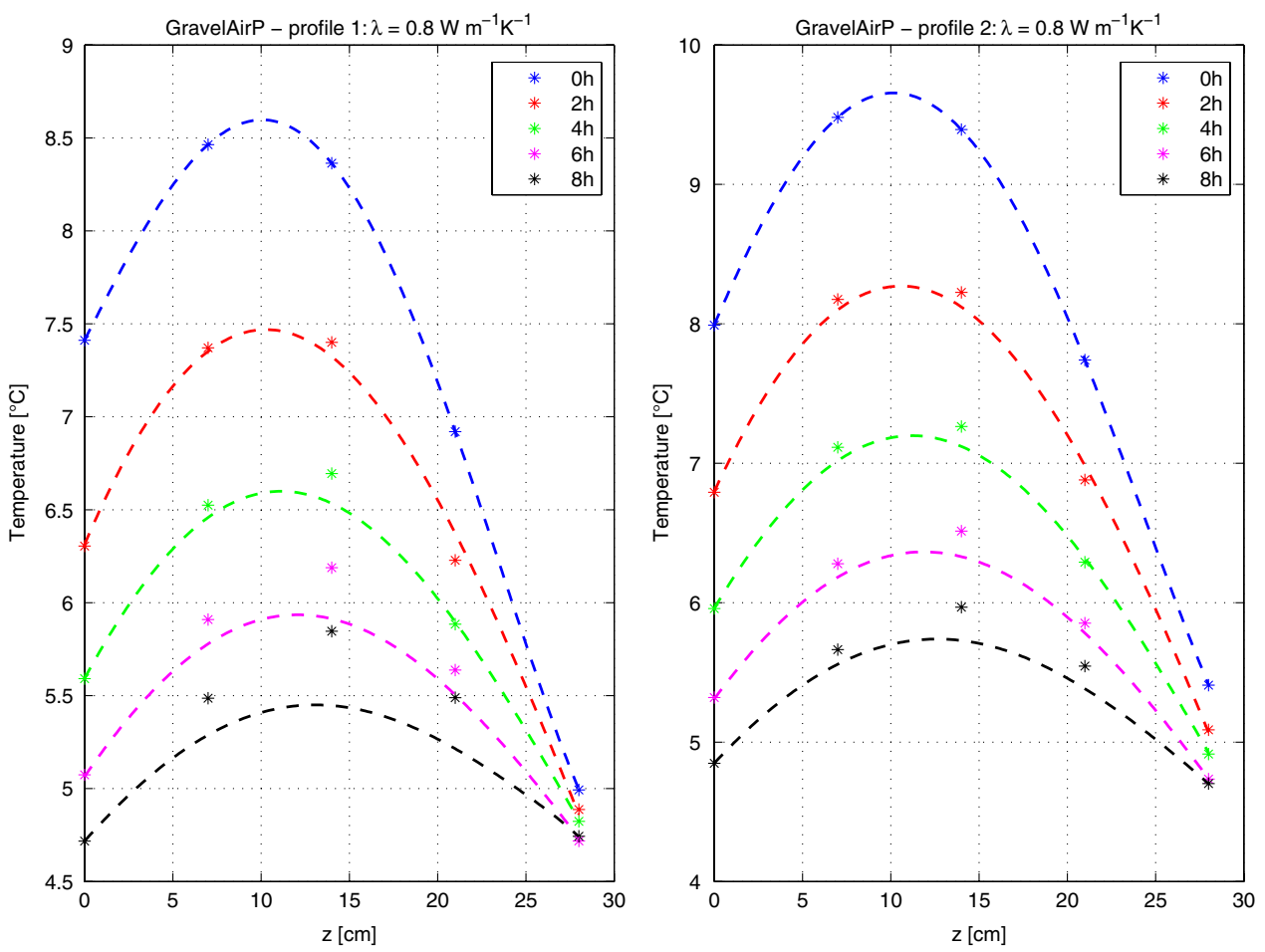

Fig. 11 Fitting of the measured temperature profiles inside the gravel sample by a heat conduction model. The two panels correspond to the temperature profile measured along the vertical paths indicated in Fig. $8 . z=0 \mathrm{~cm}$ corresponds to the bottom of the sample, $z=28 \mathrm{~cm}$ is the position of the uppermost sensors, which are placed a few $\mathrm{cm}$ below the sample surface 
crease. We suppose that these deviations are due to lateral heat fluxes (deviations from 1D-geometry) which may develop more easily in such a large-grained porous sample. The cold air from above has much easier access to the sample interior than in the case of a fine-grained sand sample. The assumed thermal insolation of the lateral boundaries may also become violated during thermal evolution.

\subsection{EXTASE probe measurements}

The EXTASE probe has two principal functions: passive temperature sensing along its $32 \mathrm{~cm}$ length, and active heating of selected sensors. In contrast to the needle probes where a long part of the thin needle is homogeneously heated during a measurement, the EXTASE probes consist of sensors with different lengths and positions along the probe [11,
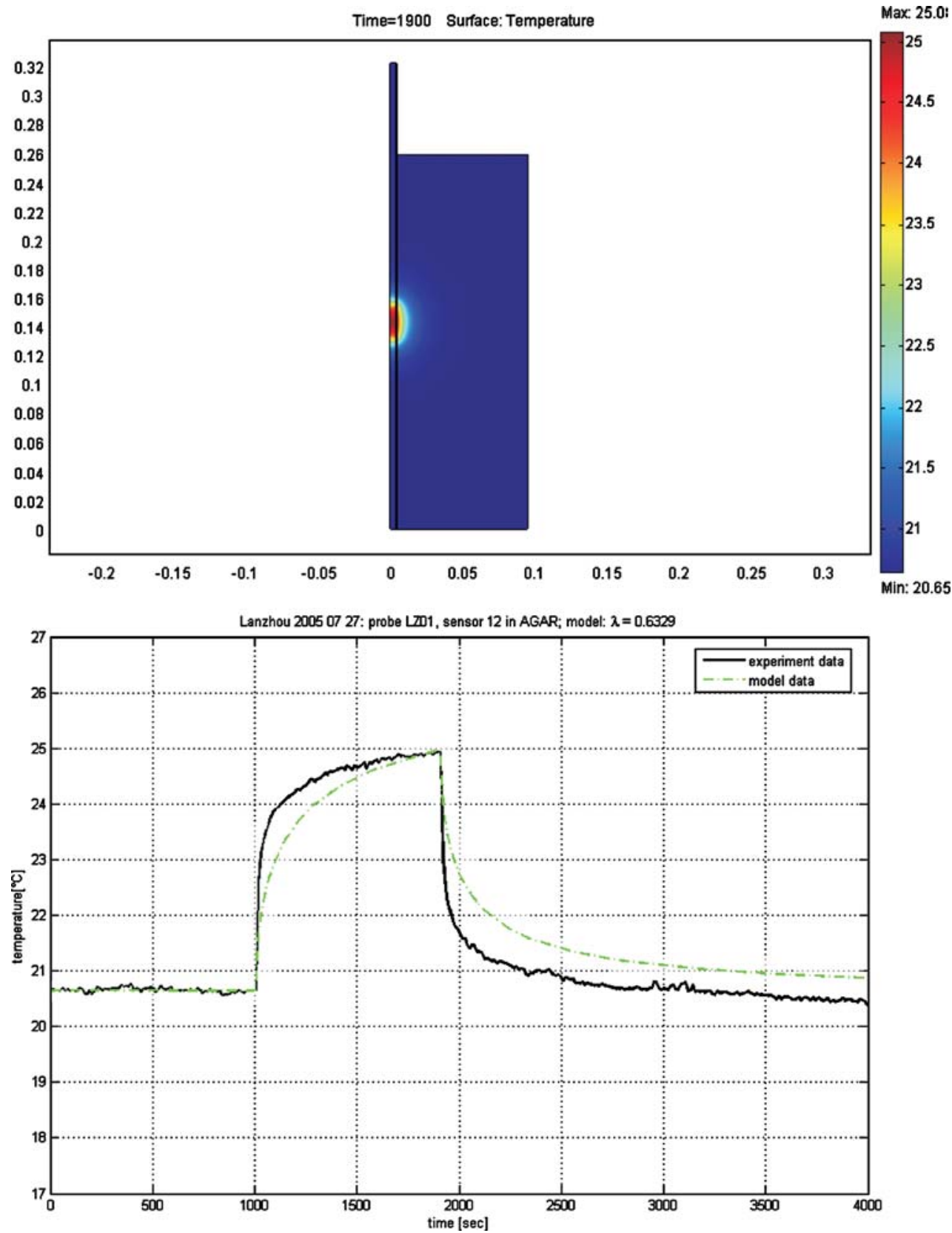

Fig. 12 Heating of EXTASE sensor $12 \mathrm{in}$ an isothermal agar-agar sample compared to a model curve with $\lambda=0.63 \mathrm{~W} \mathrm{~m}^{-1}$ as measured by the Hukseflux TP02 needle probes 
13]. In contrast to the needle probes, the single segments of the EXTASE probe violate the geometric condition that the length of the heater is large as compared to its diameter. Therefore, a thermal model using a heated cylinder with a finite height must be adopted to evaluate a value for the thermal conductivity. Strictly speaking, only the thermal diffusivity $\kappa=\frac{\lambda}{\rho c_{p}}$ can be measured, because in this case $\rho$ and $c_{p}$ does not cancel out as in the case of the needle probe. The advantage of the EXTASE probe is that when the single segments are heated sequentially, the temperature response of the heated and the neighbouring sensors can give some information on the depth profile of the thermal diffusivity.

To illustrate the performance of the probe in a wellknown medium, we show the result of a test on 27-07-2005 in agar-agar at a temperature of $17^{\circ} \mathrm{C}$. In this test, several high quality measurements with the Hukseflux needle probes were made before, which gave a value of $\lambda=0.63$ $\mathrm{W} \mathrm{m}{ }^{-1} \mathrm{~K}^{-1}$. This value was used in the thermal model simulating the heating history of the different EXTASE sensors which were in contact with the medium. As the sample was not deep enough to cover the whole probe, only the sensors 8-16 were immersed in the agar-agar. The total height of the sample was $26 \mathrm{~cm}$. The heating time for each sensor was $15 \mathrm{~min}$ followed by a cooling phase. The total duration of the test was $8 \mathrm{~h} 15 \mathrm{~min}$.

In Fig. 12, the response of the sample to heating of sensor 12 (positioned close to the center of the sample) is illustrated. The upper panel shows the temperature distribution in the whole sample after sensor 12 was heated for 15 min with a power of $0.37 \mathrm{~W}$. In the lower panel, the temperature response of sensor 12 is plotted together with a calculated temperature profile where $\lambda=0.63 \mathrm{~W} \mathrm{~m}^{-1} \mathrm{~K}^{-1}$ was used as input parameter. As can be seen from the figure, the total height of the temperature increase during the heating period fits quite well, but the speed of the temperature increase (and also the subsequent decline phase) is underestimated by the model. Generally it was observed that the coincidence is best for the slender sensors positioned close to the middle of the sample, while closer to the surface the amplitude of the model curves is $10-20 \%$ below the measured value. The faster temperature increase of the sensor at the start of the heating period and the faster cooldown after heating is completed may have different reasons. One may be the complicated fine structure of the tube (glass fiber, kapton, gluing material and conductors), which has not been modelled in full detail. Another reason can be poor thermal contact to the medium which would block the heat flow into the medium in the initial phase. This is, however, improbable in this case, as the agar gel encloses the probe tightly. Clearly, further tests and modelling efforts are necessary before EXTASE type probes can be used for measuring thermal diffusivity profiles on a routine basis.

\section{Conclusions}

- The three different methods used to determine the thermal conductivity of the laboratory samples gave by and large consistent results.

- In the sandy materials investigated, the moisture content of the samples has a strong influence on the thermal conductivity, typically with a factor of two between completely dry sand and sand with a few percent moisture content.

- Measurements in the blocky stones gravel have to be considered with care, because the grain size is of the order of the sensor size. The conductivity obtained from the measurements with the hot wire sensors is consistent with the conductivity derived from a thermal model which uses the cooling phases of the chamber and the sample bottom plate as boundary conditions. Both measurements give average values around $0.8 \mathrm{~W} \mathrm{~m}^{-1}$ $\mathrm{K}^{-1}$ which indicates that there is little influence of air convection inside the stones on the effective thermal conductivity.

- The measurements with the EXTASE/MUPUS type probes also give consistent results in simple materials like agar samples. However, interpretation in terms of thermal modelling is difficult and more calibration tests are necessary to determine the thermal response of the sensor itself more closely.

Acknowledgments This work was supported in part by the Austrian Fonds zur Förderung der wissenschaftlichen Forschung under project L317-N14. We also thank the Chinese and the Austrian Academy of Sciences and the CAREERI for their support.

\section{References}

1. Biele J (2002) The experiments onboard the Rosetta Lander. Earth Moon Planets 90:445-458

2. Carslaw HS, Jaeger JC (1959, reprinted 1980) Conduction of heat in solids. Oxford University Press, New York, pp 510

3. Espinasse S (1984) Modelisation du comportement thermique et de la differenciation chimique des noyaux de cometes. Ph.DThesis, CNRS Laboratoire de Glaciologie et Geophysique de L'Environment, Grenoble, pp 223

4. Espinasse S, Klinger J, Ritz C, Schmitt B (1991) Modeling of the thermal behavior and of the chemical differentiation of cometary nuclei. Icarus 92:350-365

5. Evitherm (2007) http://www.evitherm.org/default.asp?ID=308

6. Grigull U, Sandner H (1986) Wärme- und Stoffübertragung, Springer, Heidelberg

7. Hukseflux Thermal Sensors (2003) TP02—non-steady-state probe for thermal conductivity measurement: USER MANUAL, 47 pages

8. Kömle NI, Kargl G, Seiferlin K, Marczewski W (2002) Measuring thermo-mechanical properties of cometary surfaces: in situ methods. Earth Moon Planets 90:269-282 
9. Kosky JA, McVey DF (1986) Application of parameter estimation techniques to thermal conductivity probe data reduction. Thermal conductivity 17, Plenum Press, New York, 587-600

10. Marczewski W, Schröer K, Seiferlin K, Usowicz B, Banaszkiewicz M, Hlond M, Grygorczuk J, Gadomski S., Krasowski J, Gregorczyk W, Kargl G, Hagermann A, Ball AJ, Kührt E, Knollenberg J, Spohn T (2004) Prelaunch performance evaluation of the cometary experiment MUPUS TP. J Geophys Res 109(E7):1-17

11. Schröer K (2006) Eine kompakte Sonde für Temperatur- und Wärmeleitungsmessungen in den Geowissenschaften. Ph.D-Thesis, Westfälische Wilhelms-Universität Münster, p 203

12. Seiferlin K, Kömle NI, Kargl G, Spohn T (1996): Line heat source measurements of the thermal conductivity of porous $\mathrm{H}_{2} \mathrm{O}$ - ice, $\mathrm{CO}_{2}$-ice and mineral powders under space conditions. Planet Space Sci 44:691-704

13. Spohn T, Seiferlin K, Hagermann A, Knollenberg J, Ball AJ, Banaszkiewicz M, Benkhoff J, Gadomski S, Grygorczuk J, Hlond M, Kargl G, Kührt E, Kömle N, Marczewski W, Zarnecki JC (2007) MUPUS - a thermal and mechanical properties probe for the Rosetta Lander PHILAE. Space Sci Rev 128:339-362. doi:10.1007/s11214-006-9081-2

14. Feng WJ, Ma W, Zhang LX (2006) Application of awning to roadway engineering on the Qinghai-Tibet plateau. Cold Regions Science and Technology 45:51-58

15. Wen Z, Sheng Y, Ma W, Deng JS (2005) Experimental research on thermal conductivity of undisturbed frozen samples from permafrost regions on Qinghai-Tibetan plateau. Journal of Glaciology and Geocryology 27(2):182-187 